\title{
A Fast Semiautomatic Algorithm for Centerline-Based Vocal Tract Segmentation
}

\author{
Anton A. Poznyakovskiy, ${ }^{1}$ Alexander Mainka, ${ }^{2,3}$ Ivan Platzek, ${ }^{4}$ and Dirk Mürbe ${ }^{2,3}$ \\ ${ }^{1}$ Department of Otorhinolaryngology, University Hospital Carl Gustav Carus, Technische Universität Dresden, \\ 01062 Dresden, Germany \\ ${ }^{2}$ Division of Phoniatrics and Audiology, Department of Otorhinolaryngology, University Hospital Carl Gustav Carus, \\ Technische Universität Dresden, 01062 Dresden, Germany \\ ${ }^{3}$ Voice Research Laboratory, Hochschule für Musik Carl Maria von Weber, 01067 Dresden, Germany \\ ${ }^{4}$ Department of Radiology, University Hospital Carl Gustav Carus, Technische Universität Dresden, 01062 Dresden, Germany \\ Correspondence should be addressed to Anton A. Poznyakovskiy; anton.poznyakovskiy@uniklinikum-dresden.de
}

Received 23 January 2015; Accepted 8 April 2015

Academic Editor: Haldun Oguz

Copyright (C) 2015 Anton A. Poznyakovskiy et al. This is an open access article distributed under the Creative Commons Attribution License, which permits unrestricted use, distribution, and reproduction in any medium, provided the original work is properly cited.

\begin{abstract}
Vocal tract morphology is an important factor in voice production. Its analysis has potential implications for educational matters as well as medical issues like voice therapy. The knowledge of the complex adjustments in the spatial geometry of the vocal tract during phonation is still limited. For a major part, this is due to difficulties in acquiring geometry data of the vocal tract in the process of voice production. In this study, a centerline-based segmentation method using active contours was introduced to extract the geometry data of the vocal tract obtained with MRI during sustained vowel phonation. The applied semiautomatic algorithm was found to be time- and interaction-efficient and allowed performing various three-dimensional measurements on the resulting model. The method is suitable for an improved detailed analysis of the vocal tract morphology during speech or singing which might give some insights into the underlying mechanical processes.
\end{abstract}

\section{Introduction}

The process of human voice production involves a complex interaction of different components and mechanisms. It involves the generation of a pulsating transglottal airflow which is filtered by the vocal tract (VT) resonator. The shape of the VT, the aeroacoustic cavity between the vocal folds and the lips, defines the formant frequencies and the frequency response of the filter which, in turn, defines vowels, consonants, and essential parts of voice timbre [1].

Magnetic resonance imaging (MRI) has become a promising technique for investigating the $\mathrm{VT}$ at a functional stage. MRI delivers images of high spatial resolution which can be analyzed in two dimensions within a single sagittal plane allowing for detailed analysis of dynamic VT adjustments during speech or even singing. For a detailed overview, see [2].
The elongated structure of the VT along with its curved shape makes segmentation feasible with the use of centerlinebased methods. In medical imaging, these approaches have been successfully used in vessel segmentation [3]. The image stack is transformed into a coordinate system which is aligned with the centerline, and the cross sections are segmented. This makes a reduction of the $3 \mathrm{D}$ segmentation problem to a set of two-dimensional problems possible.

In many cases, the estimation of the centerline is a problem in itself, as in the aforementioned vessel segmentation task. There exist a broad variety of segmentation methods which compute the centerline on runtime, based on segmentation results of previous cross sections. Some of them postulate a circular [4] or else analytically defined [5] crosssection shape. Other authors make use of deformable models, subsequently skeletonizing the shape and extrapolating the resulting centerline, such as Li and Ourselin [6]. In our recent study on the cochlea [7], we also used deformable models in 
form of active contours [8] but predicted the centerline via mass centers of cross sections with the help of a Kalman filter [9].

With regard to segmentation, the geometrical structure of the VT poses similar challenges as vessel or cochlear segmentation. The potential of tomographic imaging techniques, especially MRI, to deliver three-dimensional (3D) image stacks of the VT has been exploited, for instance, to analyze area functions during sustained phonation $[10,11]$. So far though, applications to the VT mostly included manual segmentation or relatively simple segmentation algorithms. Although publications on 3D modeling of the VT exist for quite some time $[12,13]$, they rely on manual segmentation.

A few centerline-based methods have been applied to the VT. However, most studies focus solely on the extraction of the area function by employing 2D methods such as threshold segmentation $[14,15]$. A 3D centerline-based approach was presented by Vampola et al. [16], yet it still suggests segmenting individual cross sections manually. While manual segmentation requires little implementation error and is thereby a good tool for exploratory analysis, it has the drawback of being time-consuming, a factor that precludes its application to large sets of individual data.

There are several studies published which use region growing for the segmentation of VT $[17,18]$. This method does not allow for the immediate studying of VT cross sections as it lacks a centerline. In order to measure crosssectional areas, the centerline has to be constructed a posteriori, and the resulting segmented body cut along this centerline. This process might be nontrivial if there are bifurcations along the path. Indeed, VT has minor bifurcations: the piriform sinuses (sinus piriformes) and vallecula. On the other hand, vocal tract cross-sectional area has attracted considerable interest within the research community [19-23], since it plays an important role in the acoustics of speech and singing. Functional VT adjustments during phonation seem to be of importance not only for educational purposes but also for the medical field, where voice problems, for example, among professional voice users continue to bring about considerable socioeconomic burdens for the health care systems [24, 25].

Thus, in this study, we attempt to develop a VT segmentation algorithm which satisfies the following: (1) reduced operator interaction and time efforts and (2) direct data output on both VT cross sections and 3D geometry.

\section{Materials and Methods}

2.1. Image Data Acquisition and Sound Recording. A 43-yearold male test subject (height: $1.90 \mathrm{~m}$, weight: $108 \mathrm{~kg}$ ) was asked to produce a sustained vowel in a 3.0-T MR system (Verio; Siemens Medical Solutions, Erlangen, Germany) and to keep articulation constant during the recording. The task was specified regarding vowel quality (closed midback rounded vowel /o/ as in German "Boot"), pitch $(220 \mathrm{~Hz} / \mathrm{A} 3)$, and phonatory condition (speaking voice). The MRI recording was initiated as soon as the subject had started phonation. The MRI was performed with a 12-element head-neck coil. The applied MRI sequence was a volumetric interpolated breath-hold examination sequence with an acquisition time of about $12 \mathrm{~s}$. A set of 52 sagittal slices of the whole VT was obtained. The parameter setting was the following: slice thickness $1.8 \mathrm{~mm}$, repetition time $4.01 \mathrm{~ms}$, echo time $1.22 \mathrm{~ms}$, matrix $288 \times 288$, field of view $300 \times 300 \mathrm{~mm}$, and flip angle $9^{\circ}$. The obtained resolution of the images was $1.04 \mathrm{~mm}$. Due to the known limitations of the MRI to visualize structures with low water content, the teeth were not detected in the MRI scan. For the segmentation of the oral cavity the segments were forced manually to remain between the tongue and the maxillary bone leaving out the space of the teeth.

An optical microphone unit (MO 2000 from Sennheiser) and a laptop PC running Audacity software (Dominic Mazzoni et al., http://audacity.sourceforge.net/, retrieved on January 20, 2015) were used for sound recording within the MRI facility. The acoustical recording was used to ensure vowel quality and pitch correctness.

2.2. Processing of Images and Coordinate Transform. The 52 sagittal images were stacked and scaled by a factor of 3.0 with ImageJ (National Institutes of Health, Bethesda, MD, USA) resulting in 156 images with a pixel size of $0.35 \mathrm{~mm}$. This scaling was necessary to facilitate the later segmentation. Then, the images were resliced to the coronal view in order to fit the distance between slices to $0.35 \mathrm{~mm}$ and to obtain uniformly sized voxels. The reslice was repeated a second time with default settings to obtain sagittally oriented images.

For further image processing, the used algorithms were implemented in our software IPTools (freeware: http://www .uniklinikum-dresden.de/das-klinikum/kliniken-polikliniken-institute/hno/forschung/forschungslabor-gehor/links, last inspected January 20, 2015). In order to increase the grayscale gradient at the air-tissue border of the VT, the image stacks were filtered using anisotropic diffusion [26].

On the midsagittal image of the stack, the centerline was drawn (see Figure 1). This was done by defining node points such that the centerline intersects the tip of the uvula and the crossing of ventricular folds and the arytenoids. This procedure was established to ensure repeatability for application to other subjects while keeping the orientation of transformed slices near-orthogonal to the pharyngeal axis. Moreover, this provides a near-parallel slicing of the ventricular folds which is essential for calculating the area function in this region.

The centerline was piecewise interpolated between these nodes using cubic splines:

$$
\mathbf{c}_{i}\left(t_{c}\right)=\mathbf{a}_{i, 3} t^{3}+\mathbf{a}_{i, 2} t^{2}+\mathbf{a}_{i, 1} t^{1}+\mathbf{a}_{i, 0},
$$

with $\mathbf{c}_{i}=\left[c_{i, x}, c_{i, y}, c_{i, z}\right]^{\mathrm{T}}$ and $t_{c} \in\{0, \ldots, 1\}$. The four coefficients were defined by setting the positions at $t=0$ and $t=1$ to the coordinates of the nodes and equalizing the first derivatives $\mathrm{d} \mathbf{v} / \mathrm{d} t$ at these positions with adjacent spline segments.

Subsequently, the image stack was transformed along the curve with a fixed spacing of 1 pixel between new images resulting in a distance of 1.04 millimeters between centers of images. The center of each image of the new stack was set at the respective position on $\mathbf{c}\left(t_{c}\right)$. The coordinate axes were set 


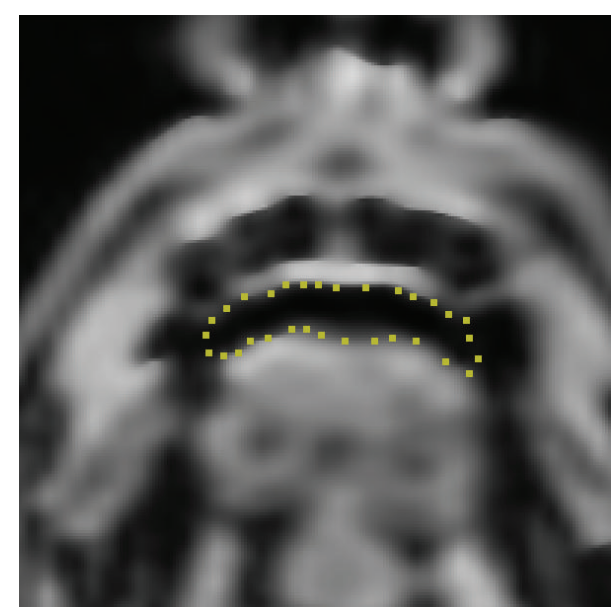

(a)

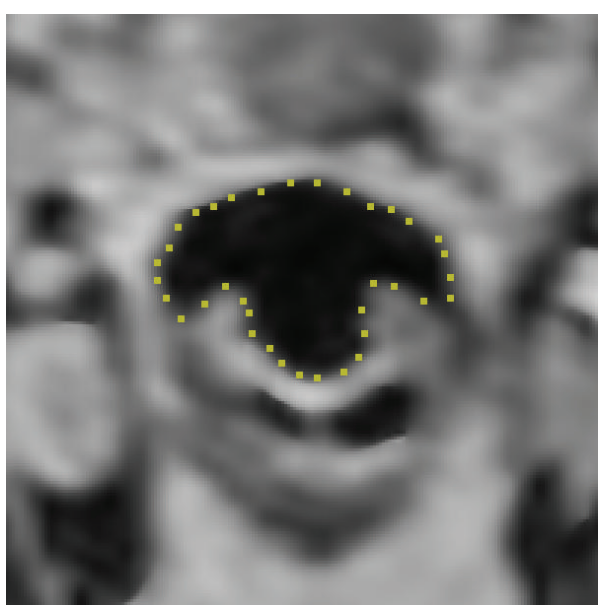

(c)

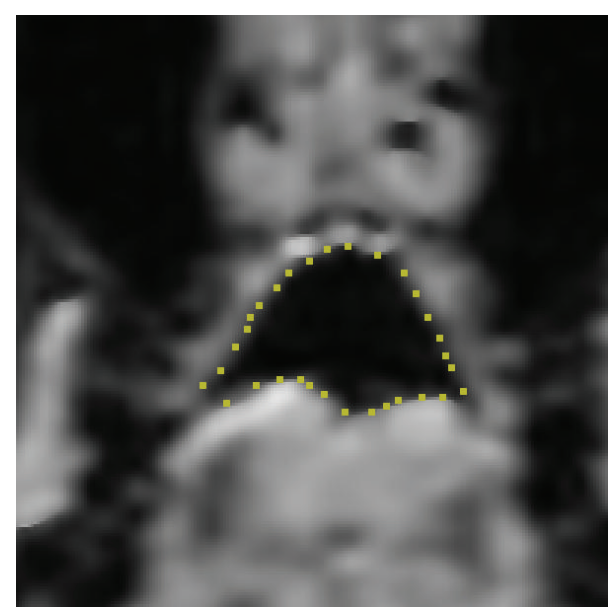

(b)

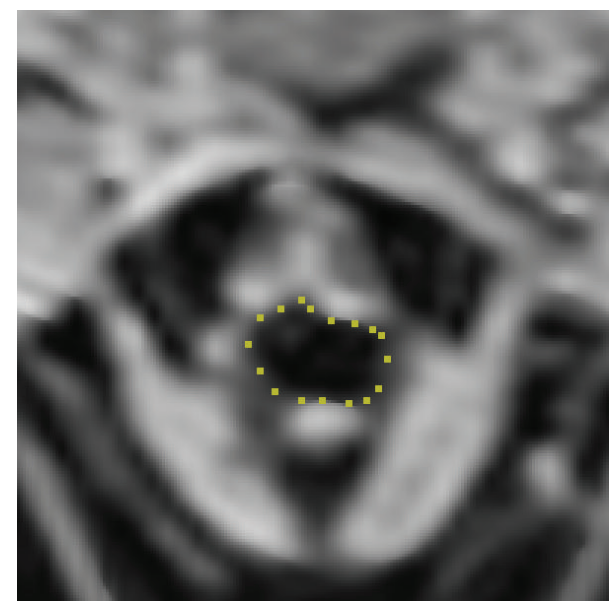

(d)

Figure 1: Four examples of vocal tract cross sections at different levels: (a) anterior oral cavity, (b) central oral cavity, (c) hypopharynx at inferior vallecula, and (d) larynx.

to the Frenet vectors of the curve at this position; specifically, the $x$-axis was set to the normalized binormal vector $\mathbf{b}$, the $y$-axis to the negated normalized normal vector $-\mathbf{n}$, and the $z$-axis to the normalized tangent vector $\mathbf{t}$. The negation of the normal vector was necessary since the coordinate system of an image stack was defined as left-hand.

From these definitions, we could derive the following affine transform matrix for each $t_{c}$ :

$$
\mathbf{T}=\left[\begin{array}{cccc}
b_{x} & b_{y} & b_{z} & c_{x} \\
\ddot{-} c_{x} & \ddot{c}_{y} & \ddot{c}_{y} & c_{y} \\
\dot{c}_{x} & \dot{c}_{y} & \dot{c}_{z} & c_{z} \\
0 & 0 & 0 & 1
\end{array}\right] .
$$

Note that the binormal vector $\mathbf{b}=\left[b_{x}, b_{y}, b_{z}\right]^{\mathrm{T}}$ is defined as

$$
\mathbf{b}=\dot{\mathbf{c}} \times \ddot{\mathbf{c}}
$$

The transformation with a set of such matrices delivered a stack of several hundred images, where each image displayed the cross section in a manner feasible for $2 \mathrm{D}$ segmentation.
2.3. Segmentation. The segmentation was performed with a greedy variant of active contours [8]. A circular discrete starting contour was initialized with a center at user-defined position. Each node of the contour had the energy balance

$$
E=E_{\text {cont }}+E_{\text {curv }}+E_{\text {ext }}+E_{\text {dev }} \text {. }
$$

Here, $E_{\text {cont }}$ is the contour energy which controls the expansive behavior of the contour. It is defined by a first-order derivative of the active contour curve function $\mathbf{v}\left(t_{v}\right)$ :

$$
E_{\text {cont }}=\alpha \cdot\left|\nabla \mathbf{v}\left(t_{v}\right)\right|^{2} \text {. }
$$

$E_{\text {curv }}$ is the curvature energy which models the bending stiffness of the contour via the second-order derivative of the curve function:

$$
E_{\text {curv }}=\beta \cdot\left|\Delta \mathbf{v}\left(t_{v}\right)\right|^{2} .
$$

The external energy $E_{\text {ext }}$ provides the contour with edge detection and is proportional to the negated square norm of the grayscale gradient:

$$
E_{\text {ext }}=-\gamma \cdot|\nabla I(x, y)|^{2} .
$$


Finally, the deviation energy $E_{\mathrm{dev}}$ provides cross links between contours on adjacent tomogram images:

$$
E_{\mathrm{dev}}=\delta \cdot d^{4} .
$$

Here, $d$ is the distance to the nearest node of the contour on previous image. In the first contour, this energy is set to zero. The Greek letters in (5)-(8) indicate user-defined parameters.

A search for the local minimum of the energy sum over all nodes was performed. This caused the contour to expand and adapt to the cross section of the vocal tract iteratively. When the contour stopped moving, the finding of local energy minimum was stated and the algorithm moved to the next image. Alternatively, the processing of the contour on an image stopped when the number of iterations exceeded a predefined threshold $(n=30)$. On the next image, a new contour was initialized with the end result of the previous image.

As the contour expanded, new points were added between any two neighboring points, whose spacing exceeded a predefined value $s_{\max }$. Similarly, one of two points was deleted if the spacing became lower than $s_{\min }$ after any iteration. For this purpose, the values were defined as $s_{\max }=4 s_{\min }$ and $s_{\min }=3 \mathrm{px}$.

The algorithm progress through the image stack was terminated at user's command. The accuracy of the segments was checked by an experienced laryngologist and corrected manually if needed.

The resulting segment stack was realigned with the information of the centerline curvature and visualized using Amira (FEI Visualization Sciences Group, Burlington, MA, USA).

\section{Results and Discussion}

Using the abovementioned methods, we were able to segment cross sections along the entire vocal tract (Figure 1). Total time used for segmentation (excluding filtering with anisotropic diffusion) was about 90 minutes.

To estimate algorithm objectivity, we performed the segmentation including centerline positioning twice. Out of the segmented data, we computed the area functions which are plotted against each other on Figure 2. The graphs appear to be highly correspondent down to the ventricular folds, scattering only in the region of the laryngeal ventricle. We assume that this is due to phonatory vibrations which cause blurring artifacts on VT borders and decrease the precision of segmentation.

We calculated reference volumes by superposition of segment areas. The total volume of the VT was estimated at $50528 \mathrm{~mm}^{3}$. The volume of the oral cavity was $25965 \mathrm{~mm}^{3}$, the combined volume of oropharynx and hypopharynx (segments from uvula to arytenoids including the sinus piriformes) was $21900 \mathrm{~mm}^{3}$, and the volume of larynx (segments from arytenoids to glottis) was $2663 \mathrm{~mm}^{3}$.

As a method of validation, we calculated the acoustic transfer function of the VT using PRAAT (Paul Boersma and David Weenink, http://www.fon.hum.uva.nl/praat/). It is displayed in Figure 3 and shows distinct peaks which are

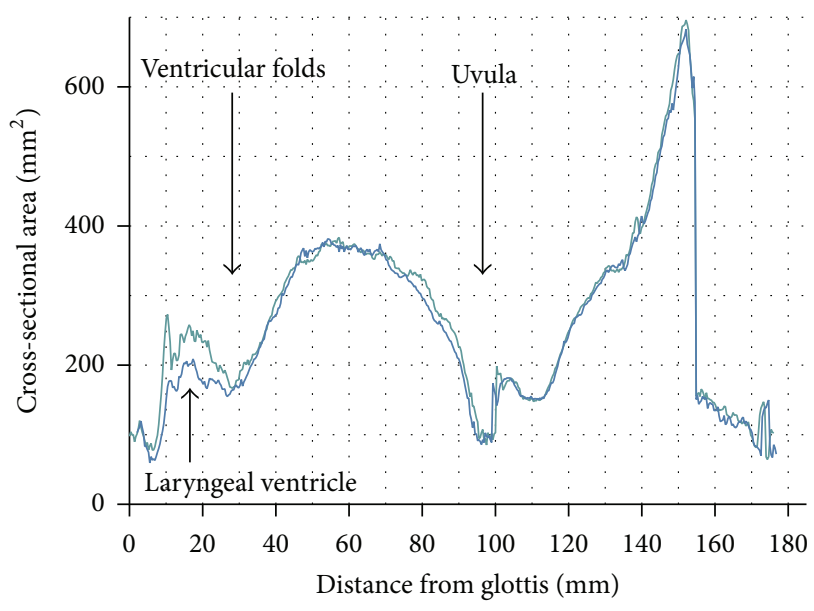

FIGURE 2: Cross-sectional area function obtained from two segmentations of the same data set.

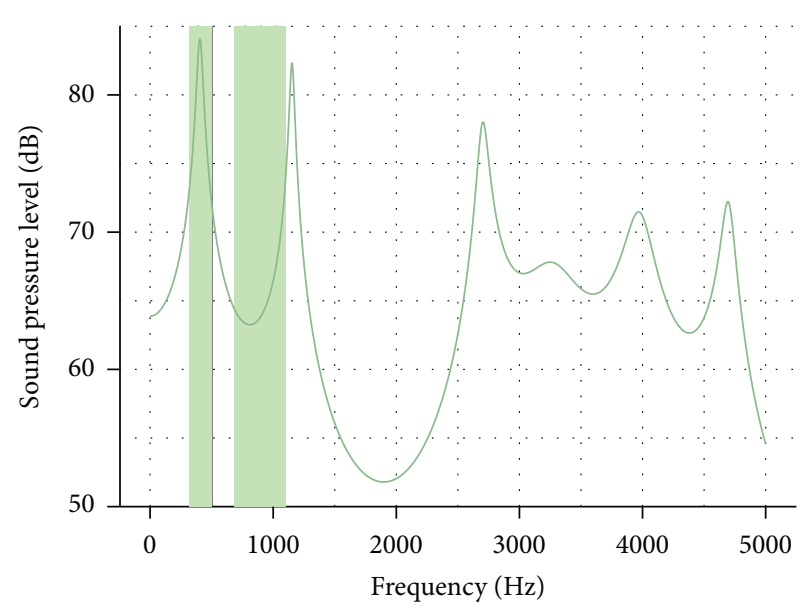

FIGURE 3: Transfer function computed with PRAAT based on the calculated area functions (Figure 2, green line). Light green stripes denote frequencies of the first two formants according to [27].

fairly coincident with the 1st and the 2nd formants for the utilized vowel [27].

With the obtained VT cross-section model, the geometrical analysis within all three spatial dimensions becomes feasible. The complete set of segmented cross sections, transformed from centerline-based coordinate system back to the global coordinate system (Figure 4(a)), is shown in Figure 4(b). Triangulation of this set yielded a surface mesh of the vocal tract shown on Figure 4(c). A close-up on the lower VT showing the high-detailed resolution of the larynx segmentation is displayed in Figure 4(d). This resulting mesh can serve as direct input for further numerical simulations using, for example, finite element modeling.

The accuracy of the model is dependent on the used MRI tomography device and the stability of the test subject over time. A natural challenge to the stability is the requirement to the subject to keep a constant articulatory setting and to maintain phonation during the entire recording procedure. This is necessary to produce enough images to 


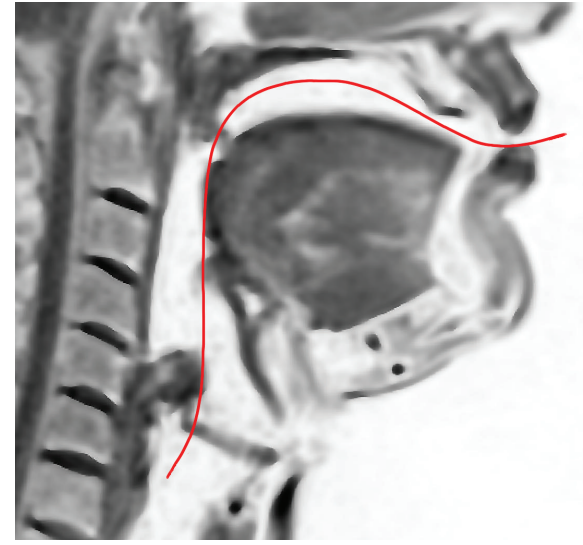

(a)

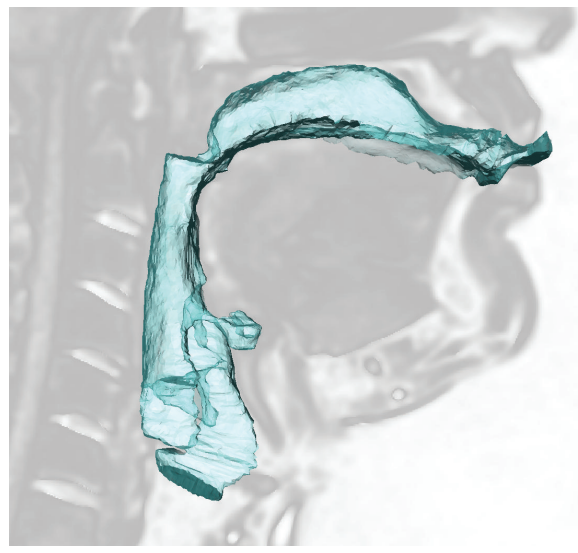

(c)

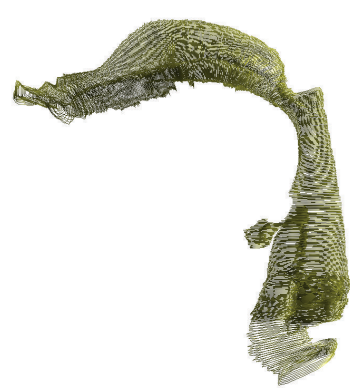

(b)

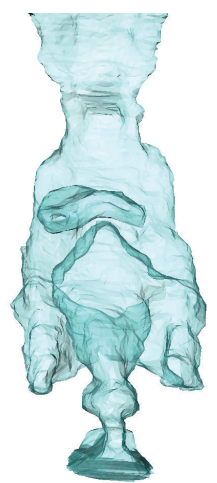

(d)

FIGURE 4: Vocal tract geometry modeling results: (a) centerline position within a midsagittal slice, (b) spatial alignment of the resulting cross-sectional segments, (c) generated surface mesh and its location within the image stack, and (d) detailed coronal view of the lower vocal tract.

cover the whole VT. There are hints that the movement artifacts of the jaw during sustained phonation are in the submillimeter order [28]. Yet this data represents only a single subject. A detailed discussion of the accuracy of MRI investigations prior to image processing is beyond the scope of this methodological study but ought to require further scientific attention.

The technical accuracy of the segmentation algorithm is constrained by the obtained resolution of MRI images. Since active contours cannot perform segmentation to a higher precision than 1 pixel, the uncertainty in border estimation corresponds to the resolution value, that is, $1.04 \mathrm{~mm}$, which is well in range of state-of-the-art publications $[15,18]$. Hence, the error in cross-section area estimation is between ca. 20 and $50 \mathrm{~mm}^{2}$, depending on the area value.

The amount of input image data calls for a time-efficient and at least semiautomatic algorithm in order to reduce manhours spent on segmentation. Unlike vessels whose crosssection shape does not vary much along the centerline, the VT has highly variable cross-section geometry (cf. Figure 1). A heuristic algorithm which is capable of handling arbitrary shapes is active contours which are widely used in biological imaging $[29,30]$. It is based on the search of a steady-state shape of discrete deformable contour under influence of an equilibrium of internal and external forces. Internal forces govern the intrinsic properties of the contour, expansion, and stiffness. The external force creates a link to the image data, attracting the contour to regions with the greatest gradient of grayscale intensity.

\section{Conclusion}

By the presented approach for a complete MRI based 3D segmentation of the VT at a functional state, we were able to obtain a high-detailed model. The method could be used for answering questions regarding the physics and mechanical properties of the VT.

\section{Conflict of Interests}

The authors declare that there is no conflict of interests regarding the publication of this paper. 


\section{Acknowledgments}

The authors acknowledge support by the German Research Foundation and the Open Access Publication Funds of the TU Dresden. The authors kindly acknowledge the help of Mario Fleischer who calculated the VT transfer function values. The authors also thank the test subject who participated in this study.

\section{References}

[1] G. Fant, Acoustic Theory of Speech Production, Mouton \& Co, The Hague, The Netherlands, 1960.

[2] M. Echternach, M. Markl, and B. Richter, "Dynamic realtime magnetic resonance imaging for the analysis of voice physiology," Current Opinion in Otolaryngology \& Head \& Neck Surgery, vol. 20, no. 6, pp. 450-457, 2012.

[3] D. Lesage, E. D. Angelini, I. Bloch, and G. Funka-Lea, "A review of $3 \mathrm{D}$ vessel lumen segmentation techniques: models, features and extraction schemes," Medical Image Analysis, vol. 13, no. 6, pp. 819-845, 2009.

[4] T. Behrens, K. Rohr, and H. S. Stiehl, "Robust segmentation of tubular structures in 3-D medical images by parametric object detection and tracking," IEEE Transactions on Systems, Man, and Cybernetics, Part B: Cybernetics, vol. 33, no. 4, pp. 554-561, 2003.

[5] P. J. Yim, J. J. Cebral, R. Mullick, H. B. Marcos, and P. L. Choyke, "Vessel surface reconstruction with a tubular deformable model," IEEE Transactions on Medical Imaging, vol. 20, no. 12, pp. 1411-1421, 2001.

[6] R. Li and S. Ourselin, "Accurate curvilinear modelling for precise measurements of tubular strcutures," in Proceedings of the 7th International Conference on Digital Image Computing: Techniques and Applications, pp. 243-252, Sydney, Australia, 2003.

[7] A. A. Poznyakovskiy, T. Zahnert, Y. Kalaidzidis et al., "A segmentation method to obtain a complete geometry model of the hearing organ," Hearing Research, vol. 282, no. 1-2, pp. 2534, 2011.

[8] M. Kass, A. Witkin, and D. Terzopoulos, "Snakes: active contour models," International Journal of Computer Vision, vol. 1, no. 4, pp. 321-331, 1988.

[9] R. E. Kalman, "A new approach to linear filtering and prediction problems," Journal of Fluids Engineering, vol. 82, no. 1, pp. 35-45, 1960.

[10] H. Takemoto, S. Adachi, T. Kitamura, P. Mokhtari, and K. Honda, "Acoustic roles of the laryngeal cavity in vocal tract resonance," The Journal of the Acoustical Society of America, vol. 120, no. 4, pp. 2228-2238, 2006.

[11] P. Clément, S. Hans, D. M. Hartl, S. Maeda, J. Vaissière, and D. Brasnu, "Vocal tract area function for vowels using three-dimensional magnetic resonance imaging. A preliminary study," Journal of Voice, vol. 21, no. 5, pp. 522-530, 2007.

[12] T. Baer, J. C. Gore, L. C. Gracco, and P. W. Nye, "Analysis of vocal tract shape and dimensions using magnetic resonance imaging: vowels," Journal of the Acoustical Society of America, vol. 90, no. 6, pp. 799-828, 1991.

[13] B. H. Story, I. R. Titze, and E. A. Hoffman, "Vocal tract area functions from magnetic resonance imaging," Journal of the Acoustical Society of America, vol. 100, no. 1, pp. 537-554, 1996.
[14] H. Takemoto, K. Honda, S. Masaki, Y. Shimada, and I. Fujimoto, "Measurement of temporal changes in vocal tract area function from 3D cine-MRI data," Journal of the Acoustical Society of America, vol. 119, no. 2, pp. 1037-1049, 2006.

[15] X. Zhou, J. Woo, M. Stone, J. L. Prince, and C. Y. Espy-Wilson, "Improved vocal tract reconstruction and modeling using an image super-resolution technique," The Journal of the Acoustical Society of America, vol. 133, no. 6, pp. EL439-EL445, 2013.

[16] T. Vampola, J. Horáček, and J. G. Švec, "FE modeling of human vocal tract acoustics. Part I: production of Czech vowels," Acta Acustica united with Acustica, vol. 94, no. 3, pp. 433-447, 2008.

[17] A. Wismueller, J. Behrends, P. Hoole, G. L. Leinsinger, M. F. Reiser, and P.-L. Westesson, "Human vocal tract analysis by in vivo 3D MRI during phonation: a complete system for imaging, quantitative modeling, and speech synthesis," Medical Image Computing and Computer-Assisted Intervention, vol. 11, no. 2, pp. 306-312, 2008.

[18] B. Delvaux, D. Howard, and D. A. Robin, "A new method to explore the spectral impact of the piriform fossae on the singing voice: benchmarking using MRI-based 3D-printed vocal tracts," PLoS ONE, vol. 9, no. 7, Article ID e102680, 2014.

[19] P. Perrier, L.-J. Boe, and R. Sock, "Vocal tract area function estimation from midsagittal dimensions with CT scans and a vocal tract cast: modeling the transition with two sets of coefficients," Journal of Speech and Hearing Research, vol. 35, no. 1, pp. 53-67, 1992.

[20] B. H. Story, I. R. Titze, and E. A. Hoffman, "Vocal tract area functions for an adult female speaker based on volumetric imaging," Journal of the Acoustical Society of America, vol. 104, no. 1, pp. 471-487, 1998.

[21] A. F. Brito, J. A. Redinz, and J. A. Plascak, "Dynamics of rough surfaces generated by two-dimensional lattice spin models," Physical Review E: Statistical, Nonlinear, and Soft Matter Physics, vol. 75, no. 4, Article ID 046106, 2007.

[22] J. Brito, "Genetic learning of vocal tract area functions for articulatory synthesis of Spanish vowels," Applied Soft Computing Journal, vol. 7, no. 3, pp. 1035-1043, 2007.

[23] Y. C. Kim, J. M. Kim, M. I. Proctor et al., "Toward automatic vocal tract area function estimation from accelerated threedimensional magnetic resonance imaging," in Proceedings of the ISCA Workshop on Speech Production in Automatic Speech Recognition (SPASR '13), Lyon, France, August 2013.

[24] K. Verdolini and L. O. Ramig, "Review: occupational risks for voice problems," Logopedics Phoniatrics Vocology, vol. 26, no. 1, pp. 37-46, 2001.

[25] R. J. Ruben, "Redefining the survival of the fittest: communication disorders in the 21st century," Laryngoscope, vol. 110, pp. 241-245, 2000.

[26] P. Perona and J. Malik, "Scale-space and edge detection using anisotropic diffusion," IEEE Transactions on Pattern Analysis and Machine Intelligence, vol. 12, no. 7, pp. 629-639, 1990.

[27] W. Sendlmeier and J. Seebode, Formantkarten des Deutschen Vokalsystems, Technical University of Berlin, Berlin , Germany, 2006, http://www.kw.tu-berlin.de/fileadmin/a01311100/ Formantkarten_des_deutschen_Vokalsystems_01.pdf.

[28] D. Aalto, J. Malinen, M. Vainio, J. Saunavaara, and P. Palo, "Estimates for the measurement and articulatory error in MRI data from sustained vowel production," in Proceedings of the 17th International Congress of Phonetic Sciences (ICPhS '11), Hong Kong, August 2011. 
[29] D. J. Kang and I. S. Kweon, "A fast and stable snake algorithm for medical images," Pattern Recognition Letters, vol. 20, no. 5, pp. 507-512, 1999.

[30] F. L. Valverde, N. Guil, and J. Muñoz, "Segmentation of vessels from mammograms using a deformable model," Computer Methods and Programs in Biomedicine, vol. 73, no. 3, pp. 233247, 2004. 


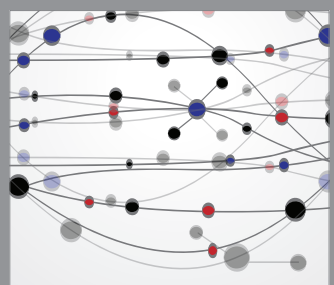

The Scientific World Journal
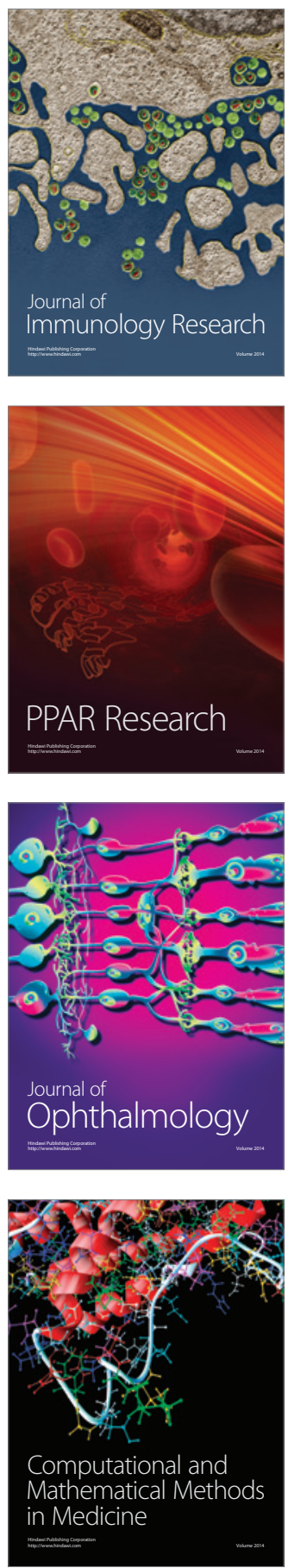

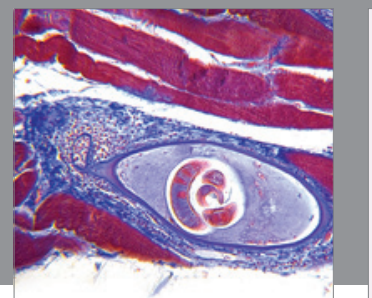

Gastroenterology

Research and Practice
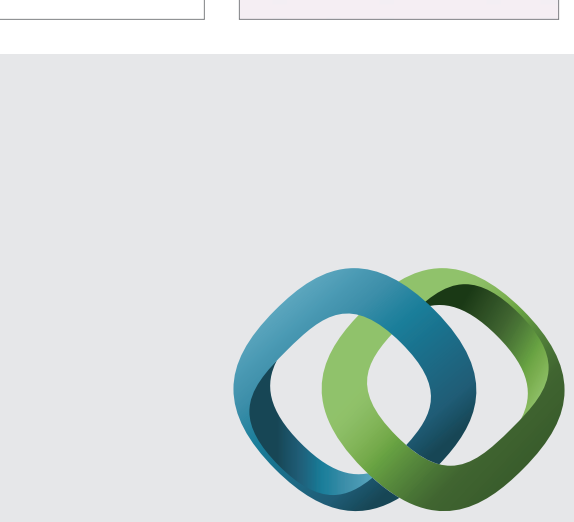

\section{Hindawi}

Submit your manuscripts at

http://www.hindawi.com
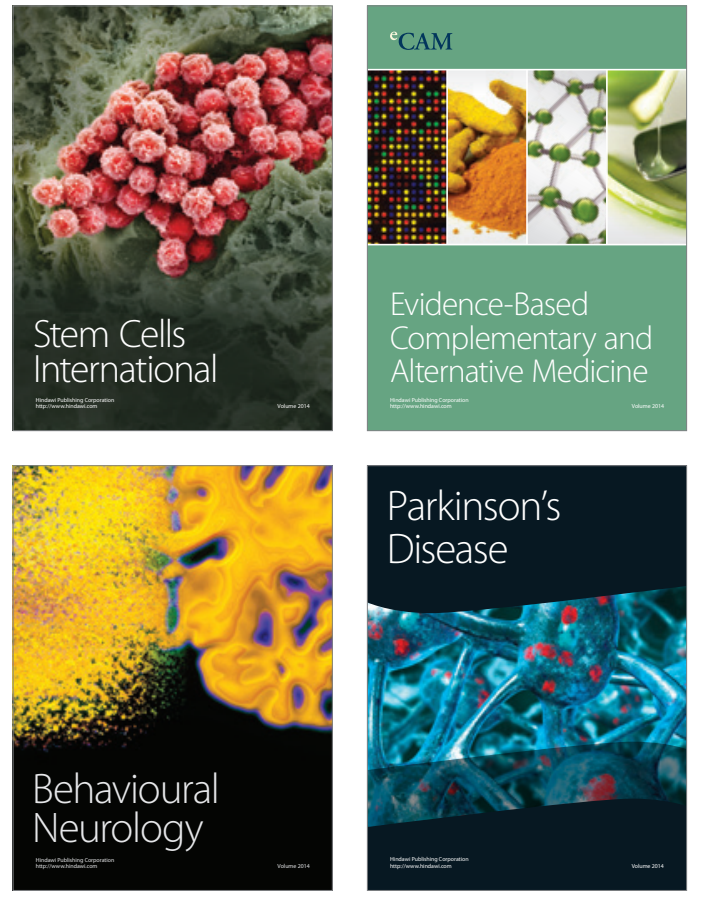
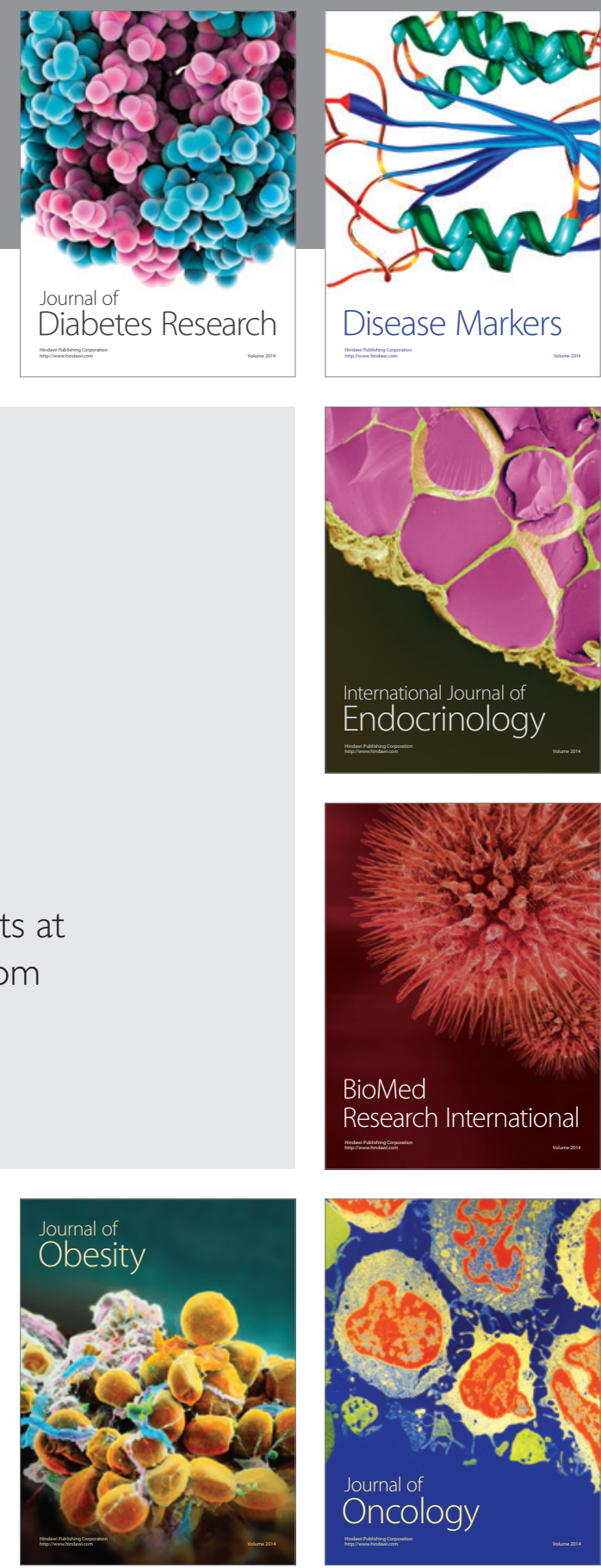

Disease Markers
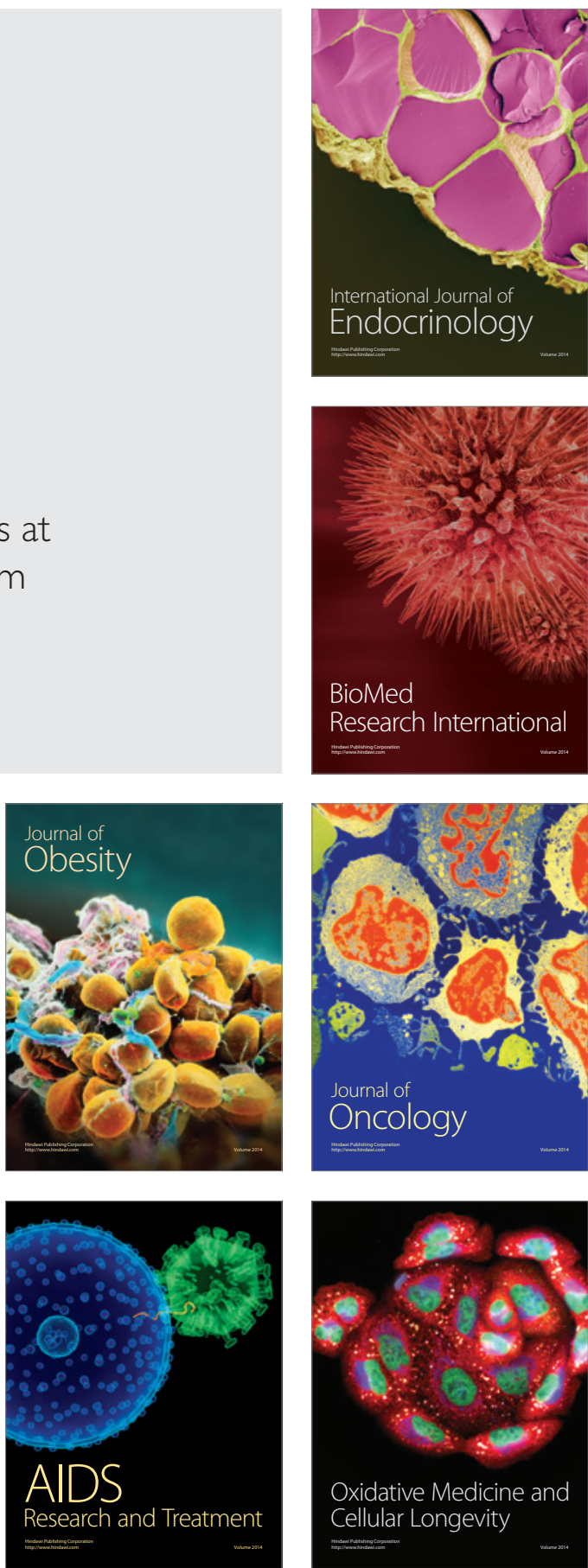